\title{
Cuidados Paliativos: Alternativa para o Cuidado Essencial no Fim da Vida
}

\author{
Railda Sabino Fernandes Alves ${ }^{1}$ \\ ${ }^{1}$ Universidade Estadual da Paraíba, PB, Brasil. \\ Gabriella Cézar Santos ${ }^{1}$ \\ ${ }^{1}$ Universidade Estadual da Paraíba, PB, Brasil.
}

\author{
Elizabeth Cristina Nascimento Cunha ${ }^{1}$ \\ ${ }^{1}$ Universidade Estadual da Paraíba, PB, Brasil. \\ Myriam Oliveira Melo ${ }^{1}$ \\ ${ }^{1}$ Universidade Estadual da Paraíba, PB, Brasil.
}

Resumo: Este trabalho apresenta uma revisão de literatura acerca dos cuidados paliativos, numa perspectiva histórico-conceitual e em interface com as políticas públicas de saúde do Sistema Único de Saúde. Cuidado Paliativo (CP) é uma abordagem que promove a qualidade de vida de pacientes e seus familiares, que enfrentam doenças que ameacem a continuidade da vida, através da prevenção e do alívio do sofrimento. CP requer a identificação precoce, avaliação e tratamento da dor e outros problemas de natureza física, psicossocial e espiritual. Este trabalho objetiva provocar uma reflexão sobre a temática dos CP, contribuindo para o estudo, aprofundamento e disseminação desse tema nos meios acadêmico, profissionais e da sociedade de um modo geral. A metodologia adotada foi uma revisão não sistematizada da literatura. Os resultados apontam para a necessidade e a urgência de maiores debates e construções teóricas sobre os $\mathrm{CP}$, indicam que existe grande lacuna na formação dos profissionais de saúde para a atuação em CP e discute a necessidade da ampliação de serviços dedicados aos CP. A propagação do assunto contribui ao debate no âmbito das políticas públicas de saúde e auxilia no processo de formação e capacitação dos profissionais para atuarem com competência e respeito à dignidade humana até os últimos momentos de vida das pessoas doentes.

Palavras-chave: Cuidados Paliativos, Humanização da Assistência, Equipe de Cuidados de Saúde, Direito a Morrer.

\section{Palliative Care: Alternative to Essential Care at the End of Life}

Abstract:This paper presents a review of the literature on palliative care in a historical-conceptual perspective and in interface with public health policies of the Unified Health System (Sistema Único de Saúde-SUS).Palliative Care is an approach that promotes the quality of life of patients and their families who face illnesses that threaten the continuity of life, through prevention and relief of suffering. Palliative Care (CP) requires the early identification, evaluation and treatment of pain and other physical, psychosocial and spiritual problems. This work aims to provoke a reflection on the theme of $\mathrm{CP}$, contributing to the study, deepening and dissemination of this theme in academic, professional and society environments in general. The methodology adopted was a non-systematized review of literature. The results point to the need and urgency of greater debates and theoretical constructions about CP, indicating that there is a great gap in the training of health professionals to work in $\mathrm{CP}$ and the need to expand services dedicated to $\mathrm{CP}$ is discussed. The spread of the subject contributes to the debate in the scope of public health policies and assists in the process of training and qualification of professionals to act with competence and respect to human dignity up to the last moments of the life of sick people.

Keywords: Palliative Care, Humanization of Assistance, Patient Care Team, Right to Die. 


\title{
Cuidados Paliativos: Alternativa para el Cuidado Esencial en el Fin de la Vida
}

\begin{abstract}
Resumen: Este trabajo presenta una revisión de literatura acerca de los cuidados paliativos, en una perspectiva histórico-conceptual y en interfaz con las políticas públicas de salud del Sistema Único de Salud (SUS). El cuidado paliativo es un enfoque que promueve la calidad de vida de pacientes y sus familiares, que enfrentan enfermedades que amenacen la continuidad de la vida, a través de la prevención y el alivio del sufrimiento. El cuidado paliativo $(\mathrm{CP})$ requiere la identificación precoz, evaluación y tratamiento del dolor y otros problemas de naturaleza física, psicosocial y espiritual. Este trabajo, objetiva provocar una reflexión sobre la temática de los CP, contribuyendo para el estudio, profundización y diseminación de ese tema en los medios académico, profesionales y de la sociedad de un modo general. La metodología adoptada fue una revisión no sistematizada de la literatura. Los resultados apuntan a la necesidad y la urgencia de mayores debates y construcciones teóricas sobre los $\mathrm{CP}$, indican que existe una gran laguna en la formación de los profesionales de salud para la actuación en CP y discute la necesidad de la ampliación de servicios dedicados a los CP. La propagación del tema contribuye al debate en el ámbito de las políticas públicas de salud y auxilia en el proceso de formación y capacitación de los profesionales para actuar con competencia y respeto a la dignidad humana hasta los últimos momentos de vida de las personas enfermas.
\end{abstract}

Palabras clave: Cuidados Paliativos, Humanización de la Asistencia, Equipo de Atención de Salud, Derecho a Morir.

\section{Introdução}

Mas tenho muito medo do morrer. O morrer pode vir acompanhado de dores, humilhações, aparelhos e tubos enfiados no meu corpo, contra a minha vontade, sem que eu nada possa fazer, porque já não sou mais dono de mim mesmo; solidão, ninguém tem coragem ou palavras para, de mãos dadas comigo, falar sobre a minha morte, medo de que a passagem seja demorada. Bom seria se, depois de anunciada, ela acontecesse de forma mansa e sem dores, longe dos hospitais, em meio às pessoas que se ama, em meio a visões de beleza. Dizem as escrituras sagradas: "Para tudo há o seu tempo. Há tempo para nascer e tempo para morrer". A morte e a vida não são contrárias. São irmãs. A reverência pela vida exige que sejamos sábios para permitir que a morte chegue quando a vida deseja ir. Cheguei a sugerir uma nova especialidade médica, simétrica à obstetrícia: a "morienterapia". O cuidado com os que estão morrendo. A missão da morienterapia seria cuidar da vida que se prepara para partir. Cuidar para que ela seja mansa, sem dores e cercada de amigos, longe de UTIs. Já encontrei a padroeira para essa nova especialidade: a "Pietà" de Michelangelo, com o Cristo morto nos seus braços. Nos braços daquela mãe o morrer deixa de causar medo (Rubem Alves, 2003).
Esse recorte, de uma das crônicas de Rubem Alves, revela que o medo de morrer é um sentimento extensamente compartilhado na sociedade atual. Muitas vezes o fenômeno da morte vem acompanhado de grande sofrimento, tanto para quem está morrendo, quanto para os que ficam. Para Rubem Alves, bom seria se a morte viesse mansa e sem dor, mas quando isso não acontecesse, o que poderia amenizar o sofrimento seria uma nova forma de cuidar nomeada pelo autor de "morienterapia", que teria a missão de cuidar da vida que se prepara para partir. Em outras palavras o autor afirma que mesmo diante da morte ainda existem cuidados que aliviam o sofrimento, proporcionam qualidade de vida e respeito à dignidade ao ser humano até os últimos momentos de sua existência. Esse tipo de cuidado anunciado pelo autor está na essência do que propõe os Cuidados Paliativos (CP), tema central deste trabalho.

Os CP, segundo a Organização Mundial de Saúde (OMS), são definidos como uma abordagem que valoriza a qualidade de vida, dos doentes e suas famílias no enfrentamento dos problemas ocasionados por doenças ameaçadoras de vida, através da prevenção e alívio do sofrimento, identificação precoce, avaliação correta e tratamento da dor e outros problemas de ordem física, psicossocial e espiritual (Word Health Organization [WHO], 2002). 
De acordo com Pessini e Bertachini (2006), os CP são aconselhados nos quadros de enfermidade avançada, progressiva e incurável; na falta de resposta ao tratamento específico; na presença de numerosos sintomas intensos, múltiplos, multifatoriais e mutantes; na presença de grande impacto emocional no doente, na família e na equipe de cuidadores, relacionado com a presença explícita ou não da morte; e prognóstico de vida inferior a seis meses.

Tratar deste tema é cada vez mais urgente, tendo em vista que com a evolução da ciência e o aumento das tecnologias em saúde, a partir da segunda metade do século $\mathrm{XX}$, a expectativa de vida das pessoas aumentou e, consequentemente, cresceu o número de pessoas longevas. Com efeito, aumentou também a quantidade de pessoas com doenças crônicas e de doentes que necessitam de CP (Alves, Melo, Andrade, \& Sousa, 2014).

Considerando a importância deste assunto, o objetivo geral deste trabalho é provocar uma reflexão sobre a temática dos CP, contribuindo para o estudo, aprofundamento e disseminação desse tema nos meios acadêmico, profissionais e da sociedade de um modo geral. E os objetivos específicos: proporcionar um conhecimento sobre a história e conceitos acerca dos CP; oferecer informações sobre programas e políticas públicas do Sistema Único de Saúde (SUS) voltados aos CP; apresentar o papel e atuação do psicólogo e da equipe multiprofissional que atuam nas práticas paliativistas; e, quiçá, contribuir para um debate crítico e reflexivo que verse sobre a desmistificação do processo de fim de vida.

Nosso intuito é fomentar novas discussões e debates que incentivem novos pesquisadores a construírem novas pesquisas; uma vez que o tema é denso, amplo e carregado de tabus e preconceitos, precisando ser disseminado cada vez mais.

\section{Método}

O procedimento metodológico adotado foi uma revisão não sistematizada da literatura. De acordo com Gil (2002) esse tipo de pesquisa traz como vantagem a possibilidade de trabalhar o tema escolhido de maneira extensa e com uma cobertura mais ampla do que aquela obtida por outros métodos. Foram realizadas pesquisas exploratórias a partir de material já publicado em livros, artigos científicos, Leis e Resoluções do SUS e de Conselho Federal de Medicina. Foram consultados também sites de associações de $\mathrm{CP}$ nacionais e internacionais.
Conforme Severino (1993), a pesquisa bibliográfica é extremamente importante e é realizada a partir de registros disponíveis decorrentes de pesquisas anteriores, em documentos impressos, livros, artigos, teses etc. E utiliza dados ou categorias teóricas já trabalhados por outros pesquisadores e devidamente registrados. Os textos tornam-se fontes a serem pesquisadas e o pesquisador pondera as contribuições necessárias à construção do trabalho.

Os materiais foram selecionados de forma abrangente, porém buscando alcançar o critério de relevância para atingirmos os objetivos da pesquisa. Por extensão, foi realizada uma análise crítica, reflexiva e interpretativa do material explorado, no intuito de construir uma argumentação que provoque uma reflexão acerca do tema apresentado.

\section{Cuidados paliativos e o moderno movimento hospice: origens e conceitos}

Ao cuidar de você no momento final da vida, quero que sinta que me importo pelo fato de você ser você, que me importo até o último momento de sua vida, e faremos tudo o que estiver ao nosso alcance não somente para ajudá-lo a morrer em paz, mas também para você viver até o dia de sua morte (Saunders, 2004).

Em princípio, o termo paliativo não esteve associado a cuidados dedicados às pessoas com doenças ameaçadoras da vida. Porém, a essência do termo revela sinais de cuidado e atenção; uma vez que, "paliativo" é derivado do latim "pallium", que significa capa, manto e está relacionado ao casaco de lã que os pastores de ovelhas utilizam para enfrentar o frio e o clima inconstante (Kovács, 1999).

O nascedouro dos CP tem suas raízes no pensamento filosófico do moderno movimento hospice, também conhecido como "assistência hospice" (Pessini, \& Bertachini, 2006). Mais do que um "ato de caridade", o moderno movimento hospice era regido por uma filosofia do cuidado, da acolhida e hospitalidade. Há indícios de que os hospices surgiram há muitos séculos na Europa como sendo lugares de acolhimento e cuidados especiais (Saunders, 2004).

Já na antiguidade, algumas referências revelam indícios de lugares onde as pessoas buscavam proteção, acolhimento e alívio para o sofrimento muito 
mais do que o objetivo de cura. Durante as Cruzadas na Idade Média, esses lugares foram se ampliando de tal modo que se tornavam casas de acolhimento e de caridade para doentes e moribundos geralmente advindos das guerras (Carvalho, \& Parsons, 2012). Também durante a Idade Média, por volta do século IV, alguns estabelecimentos, dirigidos por religiosos, albergavam cristãos em peregrinação para que eles recuperassem suas forças e seguissem com sua jornada (Saunders, 2004).

Com a expansão da filosofia do movimento hospice, esses lugares de acolhida e cuidados foram deixando o aspecto de caridade e assumindo uma caracterização hospitalar, originando os hospices. Tal expansão contribuiu a que o moderno movimento hospice resplandecesse, incorporando a missão de acolher, cuidar e tratar os doentes e moribundos, resguardando-lhes a dignidade na vida e na morte. $\mathrm{Na}$ atualidade os hospices, de acordo com Maciel (2012), podem ser definidos como um local, uma instituição de média complexidade, onde a filosofia dos CP é praticada com protagonismo.

A inglesa Cicely Saunders, assistente social, enfermeira e médica, teve uma participação substancial para a ampliação do movimento hospice. E um dos pontos de partida para que Saunders assumisse o compromisso com essa nova forma de cuidar foi sua relação de cuidado com David Tasma, um judeu de 40 anos, morador do Gueto de Varsóvia, com diagnóstico de carcinoma retal inoperável. Ele havia passado por uma colostomia paliativa e era atendido por Saunders, que o visitou até a sua morte. Saunders tinha com ele longas conversas e cuidados para minimização de seu sofrimento, tratando-lhe com dignidade até os últimos dias de sua vida. Por sua vez, David Tasma, mesmo com poucas condições financeiras, deixou uma pequena herança e uma carta que dizia: "Eu serei uma janela na sua Casa".

Mais do que uma janela, David Tasma se tornou um símbolo de incentivo tanto para profissionais quanto pacientes que chegavam ao St. Christopher's Hospice, criado em 1967 por Saunders. Esse hospice foi o primeiro serviço que oferecia cuidado integral ao paciente, desde controle de sintomas, alívio da dor e até atenção psicológica. Esse cuidado se dirigia para os doentes incuráveis como uma forma de resposta ativa para sanar o sofrimento dessas pessoas em busca de qualidade de vida (Floriani, \& Schramm, 2008). Desde então, o movimento hospice incorpora essa missão de acolher, cuidar e tratar a doença como uma jornada que será percorrida pelo doente e pela família/cuidador.

O St. Christopher's Hospice tornou-se um grande centro de ensino e pesquisa que permitiu a disseminação dessa nova forma de cuidar e contribuiu à consolidação dos CP. Prestava assistência integral não só aos doentes, mas também aos familiares e proporcionou um novo olhar sobre as doenças e a morte.

Os CP, no que se refere ao termo em si, foi uma criação de um médico cirurgião canadense chamado Balfour Mount, no início da década de 70 do século XX. Logo, o termo foi incorporado ao moderno movimento hospice, que antes disso utilizava a terminologia "cuidado hospice" para designar a filosofia em prática, ou seja, a clínica do cuidado propriamente dita, pautada em princípios éticos e humanos para garantir dignidade até o fim da vida (Floriani, 2009; Maciel, 2012).

Com o passar dos anos e das práticas em $\mathrm{CP}$, a OMS define, em 1990, CP como sendo o cuidado ativo e total para pacientes cuja doença não responde de modo positivo ao tratamento de cura. Objetiva proporcionar a melhor qualidade de vida possível para pacientes e familiares, através do controle da dor, de outros sintomas e de problemas psicossociais e espirituais (WHO, 1990).

Em 2002 a OMS revisa essa definição, para incluir aspectos do diagnóstico precoce da doença: CP é uma abordagem que promove a qualidade de vida de pacientes e seus familiares, com doenças ameaçadoras da continuidade da vida, através da prevenção e alívio do sofrimento. Requer a identificação diagnóstica precoce, avaliação e tratamento da dor e outros problemas de natureza física, psicossocial e espiritual (WHO, 2002).

O processo de desenvolvimento dos $\mathrm{CP}$, dos hospices à definição da OMS, vai oficializando a ideia de que mesmo diante da ameaça à continuidade de vida, a pessoa precisa ser tratada com dignidade, de modo a sentir-se aliviada dos seus sofrimentos, não só físicos, mas psíquicos e espirituais.

\section{Expansão dos hospices e da filosofia dos Cuidados Paliativos}

Conforme Floriani (2009), os CP e o moderno movimento hospice têm crescido e, após quatro décadas de seu surgimento, há registros de serviços de CP em 115 países, e em 41 países o potencial para se desenvolver e ser implantado. 
Após ter se originado na Inglaterra, por volta da década de 1960, o moderno movimento hospice foi se expandindo pelo Canadá, Estados Unidos e, já em fins do século XX, retorna a outras partes da Europa. Um dos países que se destacaram foram os Estados Unidos da América por oferecer uma grande rede de hospices, contabilizando 4.160, no ano de 2005 (Floriani, 2009).

De acordo com Floriani (2009), não existem registros seguros sobre o primeiro hospice brasileiro. Entretanto, há possibilidades de que o "Asilo da Penha" no Rio de Janeiro, fundado em 1944, por Mário Kröeff, tenha sido a primeira instituição com características de hospice, uma vez que tinha como objetivo atender pacientes pobres, com diagnóstico de câncer avançado e que não conseguiam vagas em outros hospitais.

No Brasil os CP começaram a se consolidar por volta de 1980. Em 1997 foi fundada a Associação Brasileira de Cuidados Paliativos (ABCP); em fevereiro de 2005 a Academia Nacional de Cuidados Paliativos (ANCP), juntamente com a Associação Médica Brasileira (AMB) (Carvalho, \& Parsons, 2012).

Em nosso país ainda não há total clareza quanto ao número de instituições de saúde que adotaram as diversas maneiras de trabalhar com os CP. A ANCP, com objetivo de divulgar tanto os serviços quanto os profissionais brasileiros envolvidos neste trabalho, está fazendo um mapeamento em nível nacional dos serviços de CP. Segundo o mapa que está disponível no site da ANCP (2017a), encontram-se cadastrados 136 serviços voltados aos CP para adultos e crianças, com alcance em todas as regiões do país. Este número é certamente maior, já que este levantamento ainda está sendo construído, e os serviços de CP que não estiverem cadastrados e quiserem fazer parte do mapa também podem entrar em contato pelo site da ANCP e solicitar sua inclusão.

Conforme Rodrigues (2012), atualmente, os principais serviços brasileiros em CP são: o Hospital Emílio Ribas de São Paulo (SP); o Instituto Nacional do Câncer (INCA) no Rio de Janeiro (RJ); o Hospital do Servidor Público Estadual de São Paulo (HSPE/SP); o Grupo Interdisciplinar de Suporte Terapêutico Oncológico (GISTO) do Hospital Erasto Gaertner em Curitiba (PR); o Centro de Estudos e Pesquisas Oncológicas (CEPON) de Florianópolis (SC); o Hospital do Câncer de Barretos (SP); o Hospital Costa Cavalcanti de Foz do Iguaçu (PR); o Hospital do Câncer de Londrina (PR); o Hospital do Servidor Público Municipal de São Paulo (HSPM/
SP), onde existe o Projeto Casa de Apoio-Hospedaria de Cuidados Especiais, que visa reumanizar a ética do cuidado com pacientes e familiares.

No Nordeste registra-se, na cidade do Recife, o Instituto de Medicina Integral Professor Fernando Figueira (IMIP), que em 2009 implantou um ambulatório especializado no atendimento de CP, e em 2013 inaugurou quatro programas de residências, dentre estes, um em Gerontologia e Cuidados Paliativos. Também no Recife encontram-se a Unidade de Cuidados Paliativos da UPE, e o serviço Saber Cuidar (HCP).

Registra-se, na cidade de Fortaleza, quatro serviços: duas unidades do Serviço de Cuidados Paliativos do Hospital Regional Unimed Fortaleza; o Serviço de Cuidados Paliativos do Hospital São Carlos; e a Equipe de Cuidados Paliativos do Hospital Geral de Fortaleza. Na cidade de Natal estão cadastrados atualmente dois serviços: A Liga Norte Riograndense contra o Câncer e o Hospital Monsenhor Walfredo Gurgel.

Esses serviços existentes no Brasil ainda aparecem de forma relativamente tímida. Se levarmos em consideração a dimensão territorial brasileira, o crescimento populacional e as modernas tecnologias em saúde que retardam a morte, fica patente a urgência em aumentar o número de serviços especializados em CP, que seriam os hospices, e, também ampliar a mentalidade e o preparo dos profissionais de saúde para atuar em qualquer lugar em que se façam necessários os $\mathrm{CP}$.

A criação de mais serviços dedicados aos CP é um tema que pede mais aprofundamento. Sobre isto existem posições divergentes quanto a ser uma prática realizável em qualquer serviço de saúde ou mesmo em casa do doente; ou ser uma prática que deve ser realizada num serviço específico, como num hospice, por exemplo.

No que tange à criação de serviços específicos para a prática dos CP o Conselho Federal de Medicina (CFM) criou três Resoluções que orientam nesta direção. A Resolução CFM n ${ }^{\circ}$ 1.805/2006 e a CFM n ${ }^{\circ}$ $1.931 / 2009$ tratam de orientar sobre a prática da ortotanásia e dos CP. Afirmam ser permitido ao médico, na fase terminal de enfermidades graves e incuráveis, limitar ou suspender tratamentos e procedimentos que prolonguem a vida do doente, garantindo-lhe os cuidados necessários para aliviar o sofrimento, na perspectiva de uma assistência integral, respeitando a vontade do paciente ou de seu representante legal (D’Avila, \& Ribeiro, 2011). E a Resolução CFM n ${ }^{\circ}$ 
2.156/2016 recomenda as unidades de CP como as mais adequadas para o tratamento digno e proporcional ao doente.

Na direção de que os CP podem ser realizados em qualquer lugar desde a atenção básica à alta complexidade, a ANCP produziu um documento em 2006 que apresenta os critérios de qualidade para os $\mathrm{CP}$ no Brasil, enfatizando que estes devem ser ofertados nos três níveis de atenção em saúde. Dentre os níveis é contemplado o da Atenção Básica, uma vez que ele pode ser oferecido em regime domiciliar.

Nesta mesma direção, o MS criou em 2013 o Programa Melhor em Casa que apresenta dentre seus objetivos a implementação dos CP no contexto domiciliar. O programa se justifica também como uma forma de desafogar os hospitais, ampliar o atendimento domiciliar do SUS e principalmente priorizar a busca por melhorias na qualidade de vida dos enfermos e seus familiares (Brasil, 2013).

Maciel et al. (2006), no que se refere aos que defendem os CP como filosofia aplicável em qualquer lugar, afirmam que os $\mathrm{CP}$, a despeito do conceito de hospice, se referem a uma abordagem e não a uma instituição. Cuidados paliativos são preconizados como uma abordagem integrada à atenção do doente em qualquer serviço de saúde, não requerendo um centro especializado para que possam ser implementados. Esses podem ser prestados na prática clínica em ambulatórios, consultas, hospitais, unidade de tratamento intensivo (UTI), independentemente de onde sejam necessários.

Os hospices são instituições que prestam CP, acolhem pessoas com doenças limitantes ou ameaçadoras da vida, que se encontram em estado evoluído da doença, no qual os CP são a única prática e alternativa de tratamento. Em geral, as pessoas ficam nos hospices nos últimos meses ou semanas de vida. Sendo muitas vezes pessoas que por alguma razão não podem ter esse cuidado no final da vida em casa, seja por indicação médica, por carência de estrutura para que se tenha um final de vida digno no domicílio ou por falta de cuidador e/ou familiar (Maciel, 2012).

Essas duas perspectivas, seja o hospice ou a aplicação dos CP em qualquer lugar ou serviço, na nossa opinião, não são antagônicas, já que os contextos de cuidados em saúde são cada vez mais complexos e exigem opções distintas para situações distintas. De modo que para dar conta de tantas especificidades, os dois sistemas parecem ser necessários. Este tema pede mais discussão e aprofundamento, pois ainda estamos num nível muito incipiente em termos da compreensão, tanto nos meios profissionais como nos consensuais, do que seja a filosofia dos CP e do que são os hospices.

\section{Políticas de humanização em saúde e os cuidados paliativos}

No Brasil, o tema dos CP começou a ter visibilidade através das políticas de humanização. A detecção de problemas na Rede de assistências em saúde, levou o Ministério da Saúde (MS) a trabalhar intensamente em programas e políticas nacionais de humanização (Waldow, \& Borges, 2011).

Entre 2000 e 2002, o MS inaugurou as políticas de humanização em saúde através do Programa Nacional de Humanização da Atenção Hospitalar (PNHAH) com ações em hospitais para promover a criação de comitês de humanização que buscavam a melhoria na qualidade da atenção e, também da atenção ao trabalhador; e, a humanização do parto e da saúde da criança. A $11^{\text {a }}$ Conferência Nacional de Saúde, realizada em dezembro de 2000, que teve como título "Acesso, qualidade e humanização da atenção à saúde com controle social", fortaleceu ainda mais as discussões e iniciativas para que a humanização fosse foco principal nas práticas de saúde (Pasche, \& Passos, 2008).

Contudo, diante dessas novas iniciativas em busca por humanização no Sistema de Saúde no Brasil, algumas perguntas foram inevitáveis. Por que humanizar, se as práticas já eram feitas por humanos? O que estava acontecendo para que essa nova filosofia fosse "a ordem do dia" no SUS? As respostas a estas questões vieram de levantamentos e pesquisas realizadas junto à população usuária do SUS e aos profissionais da rede de atenção de saúde, que constataram problemas principalmente no que se refere às relações e às formas como os pacientes estavam sendo tratados. Diante do levantamento o MS constatou práticas desumanas com relação a filas, à insensibilidade dos trabalhadores frente ao sofrimento das pessoas, aos tratamentos desrespeitosos, ao isolamento dos pacientes de suas redes sócio-familiares, a consultas e internações, a práticas de gestão autoritária, a degradações no ambiente e nas relações de trabalho, dentre outras coisas (Brasil, 2010).

A população reclamava da forma de atendimento, da falta de atenção e da incompreensão de 
alguns profissionais da saúde que não acolhiam satisfatoriamente suas demandas. Tais fatores pareciam ser mais valorizados do que a falta de médicos, falta de espaço nos hospitais, e até a falta de medicamentos (Brasil, 2001).

O levantamento dessa problemática teve como principal consequência a ampliação do PNHAH que passou a ser uma Política Nacional de Humanização (através do Humaniza SUS), que por meio de uma "perspectiva transversal, constitui uma política de assistência e não mais um programa específico" (Deslandes, 2004, p. 8).

A Política Nacional de Humanização da Atenção e da Gestão na Saúde (PNH), consolidada em 2003, vem para reafirmar as origens do SUS, e efetivar na concretude das práticas de saúde, os diferentes princípios do SUS (Benevides, \& Passos, 2005). Nesse sentido, para que atingisse efetivamente todo o sistema de saúde no Brasil, as iniciativas de humanização deveriam ser mais que programas aplicados isoladamente em hospitais e instituições. Devem ser compreendidas como uma política que opere transversalmente em toda a rede SUS (Brasil, 2004). Conforme Archanjo e Barros (2009), a PNH não só reafirma os princípios da universalidade, equidade e integralidade do SUS, mas, também propõe outros princípios imprescindíveis para a consolidação de um SUS resolutivo e de qualidade.

A PNH visa "ampliar o diálogo entre os sujeitos implicados no processo de produção da saúde, promovendo gestão participativa, estimulando práticas resolutivas, reforçando o conceito de clínica ampliada" (Archanjo, \& Barros, 2009, p. 2).

A política de humanização passa a ser a principal diretriz para conduzir a reflexão das ações em saúde, buscando ampliar o respeito e a qualidade das relações construídas no contexto da saúde. É a PNH que contempla e ancora os CP desde os primórdios do PNHAH, através de intervenções relativas a humanização da dor, humanização do cuidado no ambiente hospitalar, humanização da velhice, comunicação como fator de humanização na terceira idade e humanização no final da vida em pacientes idosos (Archanjo, 2010).

A filosofia da humanização das práticas de saúde não só perpassa, mas é fulcral para as assistências paliativistas. Várias situações críticas podem ser elencadas para mostrar a dimensão da necessidade de humanização das assistências de saúde no contexto dos CP: o progressivo envelhecimento populacio- nal que vem acompanhado de um predomínio de doenças crônico-degenerativas de evolução lenta; o aumento dos casos de câncer; as infecções por HIV, e outras enfermidades que comprometem o indivíduo e causam dependência (Carvalho, \& Parsons, 2012).

Com respeito ao envelhecimento e a longevidade crescente, constatamos que, muitas vezes, a longevidade vem associada a anos de sofrimento, inúmeras visitas a médicos, prolongadas internações em hospitais. Situações que trazem uma consternação física e mental para o doente, seus familiares e cuidadores.

Por um lado, não se pode negar que os avanços tecnológicos da medicina têm permitido salvar vidas de doentes críticos, com potencial de cura, mas, por outro lado, estão àqueles pacientes sem possibilidade de recuperação, em que medidas "curativas" além de onerosas e sem benefícios, acabam causando apenas mais dor e sofrimento (Lago, Devictor, Piva, \& Bergouniou, 2007).

Um dos ambientes que reflete a evolução da tecnologia são as Unidades de Terapia Intensiva, que mediante o uso máximo da ciência médica, na busca da cura das doenças e do prolongamento da vida, desconsideram o âmbito emocional e afastam o médico do vínculo com pessoas em estado grave e/ou com doenças terminais (Cohen, \& Gobetti, 2001).

Com efeito, os hospitais acabam se tornando um depositório de pacientes fora de possibilidade de cura e tratando os mesmos de forma inadequada, sempre focada na tentativa de cura, com métodos invasivos e de alta tecnologia, que muitas vezes são exagerados, insuficientes e só acarretam ainda mais sofrimento, enquanto o que essas pessoas mais desejam é aliviar a dor que sentem (Matsumoto, 2012). "Não se trata de cultivar uma postura contrária à medicina tecnológica, mas questionar a tecnolatria e refletirmos sobre a nossa conduta, diante da mortalidade humana, tentando o equilíbrio necessário entre o conhecimento científico e o humanismo, para resgatar a dignidade da vida e a possibilidade de se morrer em paz" (Matsumoto, 2012, p. 23).

Por mais que tenhamos a tecnologia científica atuando ao nosso favor, existe algo que não deve ser negligenciado, que são as relações humanas, as práticas de humanização e a filosofia do cuidado, que coloca a qualidade de vida e a dignidade humana à frente da ciência.

Questionar a tecnolatria, como referido acima, é refletir sobre os limites entre a ciência tecnológica e a 
dignidade humana, é perceber o quanto idolatramos a tecnologia por causa do medo da morte, e esquecemos que o processo de morrer é inerente ao ser humano e deve ser vivenciado por todos os indivíduos de maneira digna e concernente com suas vontades, sempre que possível.

Neste cenário, as práticas paliativistas emergem para mostrar aos profissionais que o cuidado a pessoas com doenças que ameaçam a continuidade da vida, deve ser integral. Quer dizer que ele deve ser considerado como um ser humano único, digno, que tem sua história de vida, com experiências vividas e compartilhadas entre familiares e cuidadores que se refletem, principalmente, nos momentos de dor e angústia, que deve ser respeitado até o fim. Logo, "a importância da humanização nos cuidados paliativos se faz essencial, pois esses cuidados se iniciam a partir do entendimento de que cada paciente tem sua própria história, relacionamentos, cultura e que merecem respeito, como um ser único" (Melo, 2012, p. 24).

Desse modo, a medicina paliativa inclui em seu lema principal de cuidar, auxiliar o doente a desfrutar de possíveis realizações pessoais durante o enfrentamento da doença e nos últimos momentos da vida (Pedreira, 2013).

Essa busca por práticas mais humanas é assegurada também pelos princípios da Bioética, a qual se constitui uma força aliada à abordagem dos CP. Um dos princípios da bioética propõe que a autonomia do paciente possa ser garantida através do consentimento informado, o qual possibilita que ele tome suas próprias decisões, atendendo ao princípio da beneficência e da não maleficência (Matsumoto, 2012).

O respeito e cuidado ao ser humano devem assim ser assegurados em todos os momentos de sua vida, e não é por estar em condição crítica de adoecimento, ou mesmo fora de possibilidade de cura que os cuidados essenciais e especiais devem ser esquecidos, pelo contrário, esses devem ser utilizados para que estes pacientes possam viver da melhor forma possível, até os momentos finais de vida (Pedreira, 2013).

\section{Princípios para a atuação da equipe multiprofissional nos cuidados paliativos}

De acordo com Santos (2011), os CP devem ser oferecidos por uma equipe multidisciplinar que contenha no mínimo: médicos, enfermeiros, assistentes sociais e psicólogos, que devem assegurar de modo amplo e otimista o alívio do sofrimento que a doença provoca. Em casos de doenças crônicas de alto grau de fatalidade o foco é a qualidade de vida. Não obstante, todo o cuidado deve ser oferecido logo que a doença for diagnosticada, garantindo o bem-estar físico, psicossocial e espiritual do indivíduo.

Os profissionais que buscam se capacitar em CP passam a reconhecer os limites da medicina evitando o excesso de tratamento, às vezes inútil e fútil; e aprendem a respeitar a autonomia do paciente em querer ou não dar continuidade ao tratamento (Pedreira, 2013).

Segundo Marengo, Flávio e Silva (2009, p. 355) o acompanhamento multiprofissional deve contar com a "participação de: anestesiologistas, clínicos, cirurgiões, psiquiatras, fisioterapeutas, enfermeiros, assistentes sociais e psicólogos, e muitas vezes, é necessário um apoio religioso".

O Manual de Cuidados Paliativos para Pacientes com Câncer também enfatiza a fundamental importância de uma equipe completa na prática desses cuidados para que a pessoa seja vista e tratada numa perspectiva biopsicossocial (Unidade de Cuidado [UNIC], 2009). Do mesmo modo, o Guia de Cuidados Paliativos registra a importância de uma equipe multidisciplinar humanizada no cenário dos CP (Sociedad Española de Cuidados Paliativos [SECPAL], 2011).

A Organização Mundial de Saúde (WHO, 2002) publicou uma lista dos princípios que regem a atuação da equipe multiprofissional de CP. Assim, a equipe multiprofissional deve:

1. Promover o alívio da dor e outros sintomas desagradáveis;

2. Afirmar a vida e considerar a morte como um processo normal da vida;

3. Não acelerar nem adiar a morte;

4. Integrar os aspectos psicológicos e espirituais no cuidado ao paciente;

5. Oferecer um sistema de suporte que possibilite o paciente viver tão ativamente quanto possível, até o momento da sua morte;

6. Oferecer sistema de suporte para auxiliar os familiares durante a doença do paciente e a enfrentar o luto;

7. Fazer uma abordagem multiprofissional para focar as necessidades dos pacientes e seus familiares, incluindo acompanhamento no luto;

8. Melhorar a qualidade de vida e influenciar positivamente o curso da doença;

9. Deve ser iniciado o mais precocemente possível, juntamente com outras medidas de prolonga- 
mento da vida, como a quimioterapia e a radioterapia e incluir todas as investigações necessárias para melhor compreender e controlar situações clínicas estressantes.

Nesta linha de pensamento, Matsumoto (2012) diz que o CP não deve se basear em protocolos, mas em princípios; que o termo terminalidade deve ser mudado para doença que ameaça a vida; que o cuidado deve ser iniciado desde o diagnóstico e deve se evitar falar em impossibilidade de cura. Ao invés disso, trabalhar com a possibilidade de tratamento não modificador da doença, afastando a ideia de não ter mais nada a fazer. Esta abordagem inclui a espiritualidade dentre as dimensões do ser humano e a assistência à família, após a morte do paciente, no período de luto. Nesse sentido, técnicas, protocolos e modos de fazer, parecem estar em segundo plano, quando o mais importante é olhar para o ser humano de forma integral, em sua experiência, compreendendo-o por completo naquele momento tão difícil de ser vivido.

Pessoas fora do alcance terapêutico curativo geralmente desejam estar acompanhadas de pessoas da família. É por isso que dentro dos princípios dos CP, a família também deve estar sendo assistida pela equipe multiprofissional, uma vez que os cuidadores familiares se envolvem de elevada maneira no adoecer de seu parente que começa a conviver com uma sobrecarga física, emocional, social, material, financeira e existencial, principalmente quando o curso da doença está avançado (Floriani, \& Schramm, 2006).

Mota e Martins (2006) afirmam que aceitar a morte como parte da vida ainda provoca sensações conflitantes nos profissionais de saúde. O contato direto com seres humanos, em estado de saúde ou doença, coloca o profissional de saúde diante de sua própria vida, dos próprios conflitos e frustrações. Isto, às vezes, pode levar o profissional a utilizar o distanciamento como mecanismo de defesa, devido a tensões provenientes do contato frequente com a dor e o sofrimento, o receio de cometer erros e as relações com pacientes difíceis.

É evidente a importância da formação profissional e o apoio da equipe multiprofissional entre si. "É essencial que este profissional assuma uma atitude terapêutica caracterizada pela escuta, pela compreensão das necessidades destas pessoas. Zelando e promovendo cuidado humanizado" (Pedreira, 2013, p. 11).

Esse cuidado humanizado, pautado na escuta e acolhimento, deve ser partilhado por todos os profis- sionais de saúde, entretanto, o psicólogo possui uma formação altamente especializada na escuta subjetiva do ser humano. Por isso, sua presença na equipe de CP é tão relevante, já que entre todos os profissionais de saúde é o mais preparado para lidar com questões referentes à subjetividade, podendo intervir junto à equipe, às famílias e aos pacientes, proporcionando acolhimento através de uma escuta sensível, auxiliando o sujeito a continuar sendo autônomo diante de sua vida, como ser único e digno (Melo, 2012).

\section{Atuação do psicólogo nos cuidados paliativos}

O psicólogo atua acolhendo e intervindo nas questões subjetivas do doente, dos familiares, sempre em articulação com toda a equipe, planejando as melhores formas de promover a saúde psíquica de todos os envolvidos na situação. De acordo com Pedreira (2013, p. 11) "o psicólogo direciona o olhar ao humano, priorizando um espaço de elaboração, trazendo um outro ponto de vista diferente do médico, no qual o cuidado não se reporta apenas na remissão rápida dos sintomas". O psicólogo trabalha proporcionando espaço para que o indivíduo seja e viva conforme sua vontade, como ser único que é articulado com tudo ao seu redor, inclusive com as outras pessoas.

O psicólogo oferece espaço à palavra, ao sofrimento e às angústias. Proporciona espaço para que a equipe troque opiniões, e para que a família se coloque como participante desse momento. Oferece "um lugar onde a fala possa ser e fazer sentido, singularizando a experiência do sofrimento" (Pedreira, 2013, p. 9). Essa é uma das mais importantes atribuições do psicólogo comprometido com os CP, sendo todas estas ações convergentes para a construção de um espaço em que o indivíduo viva com dignidade até os últimos momentos de vida.

Assim, ancorados na ética e nos princípios dos CP, os psicólogos devem promover espaço para que todos se envolvam em prol de uma ação educativa que viabilize a reavaliação de suas atitudes e conceitos diante da ideia de morte e procurem formas de trabalhar seus sentimentos e questões existentes relacionadas a isso (Pedreira, 2013).

Segundo o psicólogo humanista Rogers (1983), a função do psicólogo é, portanto, estabelecer a comunicação entre o paciente, a família e a equipe de saúde, facilitando a construção de espaços para escutar a todos, escutando o não dito, as metáforas, a lingua- 
gem simbólica, tudo aquilo que está nas entrelinhas do que está sendo verbalizado. Buscando essa prática, o profissional transfere o foco de sua atenção para o indivíduo e não para a doença. Portanto, o psicólogo atua como um norteador de visões e acolhedor de angústias, promovendo uma assistência psicológica humanizada (Pedreira, 2013).

Melo (2012, p. 43) propôs uma lista, intitulada "Atividades do profissional de Psicologia para intervenção nos cuidados paliativos", para nortear a prática dos profissionais de Psicologia em CP:

- Amenizar o sofrimento do paciente até sua morte;

- Melhorar a qualidade de vida do paciente, tanto no hospital, como em casa quando possível;

- Disponibilizar total assistência à família do paciente;

- Trabalhar ador emocional dos pacientes efamiliares;

- Proporcionar a busca de sua autonomia para a obtenção da dignidade do paciente;

- Atender ao desejo do paciente, quando possível;

- Entender o histórico do paciente;

- Desenvolver o apoio psicológico para a busca do bem-estar do paciente;

- Exercer as atividades em equipe multidisciplinar;

- Procurar sensibilizar a equipe de saúde que está em contato direto com o paciente fora do alcance terapêutico;

- Fazer escuta psicológica;

- Usar técnicas de esclarecimento;

- Ser o elo entre paciente/família com a equipe de saúde;

- Procurar trabalhar os processos de morrer, desde a conceituação, para melhorar a assistência aos pacientes com doenças ameaçadoras de vida;

- Em caso de óbito, acompanhar o médico no ato da comunicação do mesmo e assistir os familiares;

- Trabalhar questões espirituais, quando trazidas pelo paciente;

- Fazer um trabalho pessoal para obter uma prática humanizada;

- Buscar uma formação contínua sobre os cuidados paliativos com especializações e capacitações, para acompanhar a evolução da ciência.

Tonetto e Rech (2001) afirmam que o psicólogo nas práticas destinadas aos $\mathrm{CP}$ deve permitir que o paciente fale de si mesmo, da sua morte, ouvindo suas necessidades, além de estar disponível para tocá-lo e para aprender com ele. Deve também acolher a pessoa doente, ser continente, dar apoio, trabalhar questões espirituais, ajudar nas despedidas da vida e das pessoas que ama, buscar o apoio da família, auxiliar no seu desligamento da vida material e na aceitação da morte. Respeitar os desejos e opções e assumir postura de aceitação do inevitável são atitudes fundamentais para que a pessoa possa morrer com dignidade.

\section{Carências da formação profissional para atuação em cuidados paliativos}

A literatura sobre os CP ainda é escassa e a temática ainda desconhecida entre a maioria das pessoas, inclusive entre profissionais de saúde (Carvalho, \& Parsons, 2012). Montenegro (2012) afirma que existe uma necessidade na formação de profissionais no que se refere aos $\mathrm{CP}$ no Brasil, pois o acesso à informação ainda é restrito, tendo assim a consequência de recursos humanos escassos.

É imperante a necessidade de divulgar esta temática principalmente entre os profissionais de saúde os quais poderiam contribuir mais efetivamente para que esse cuidado seja exercido com eficácia nos ambientes de saúde. De acordo com Carvalho e Parsons (2012), a educação para esse cuidado ainda é insuficiente e muitos profissionais de saúde desconhecem técnicas de paliação.

O Ministério da Saúde reconhece como primeiro passo para que exista uma expansão dos CP no Brasil a disseminação do tema para as pessoas em geral, bem como para os profissionais de saúde. Esse é um dos pontos previstos no Programa Nacional de Cuidados Paliativos, Portaria no 19/GM/2002, no Art. $1^{\circ}$ que assume como de responsabilidade do programa, promover a educação de profissionais da saúde e da comunidade, através da articulação e promoção de iniciativas destinadas a incrementar a cultura assistencial da dor, a educação continuada de profissionais de saúde e de educação comunitária para a assistência à dor e os CP (Portaria No 19, 2002; Montenegro, 2012). A educação é uma das formas mais eficientes para criar a cultura necessária para a difusão do conceito do CP e de todas as características que lhes são inerentes (Maciel et al., 2006).

Para Floriani e Schramm (2007) e Floriani (2011), faz-se mister incluir os CP na grade curricular dos graduandos em saúde, para que os profissionais possam 
ser cada vez mais comprometidos e sensíveis às pessoas que tanto necessitam de acolhimento e atenção.

De acordo com dados da ANCP (2017b), há uma lacuna na formação de médicos e profissionais de saúde em $\mathrm{CP}$, devido à ausência de residência médica e de cursos de especialização e de pós-graduação de qualidade. Ainda hoje, no Brasil, a graduação em medicina não ensina ao médico como lidar com o paciente em fase terminal, como reconhecer os sintomas e como administrar esta situação de maneira humanizada e ativa.

Silva e Moreira (2010), em pesquisa realizada na área de enfermagem, concluíram que o profissional não tem domínio suficiente sobre a temática dos CP. Tal estudo corrobora a pesquisa realizada por Melo (2012) entre profissionais da área de Psicologia que, também demonstraram não ter conhecimento suficiente acerca dessa forma de cuidar. Ressaltaram também a falta de capacitação no que se refere aos CP e à humanização. "É necessário reformular o currículo dos cursos de saúde para que eles passem a contemplar uma visão antropológica e não apenas técnico-científica da ação na área da saúde e nos cuidados paliativos" (Barbi, 2011, pp. 23-24).

Junqueira e Kovács (2008) afirmam haver uma negação da morte nos currículos do curso de Psicologia. A negação surge como uma defesa para se evitar o contato com a própria finitude. É preciso considerar que os profissionais estarão em contato constante com a morte, nos consultórios, hospitais, escolas ou nas empresas, sendo inevitável estar melhor preparados para lidar com o tema.

Para que o psicólogo possa promover atenção e cuidado ao outro, se faz necessário que o mesmo tenha uma formação sólida e saiba lidar com suas questões pessoais, principalmente relacionadas aos processos inerentes à vida, assim como à própria morte. Crema (2001) considera grave essa lacuna na formação dos profissionais de saúde, que passam a exercer a profissão completamente despreparados, principalmente para o acompanhamento de pessoas nas fases terminais da existência.

Na pesquisa de Melo (2012), realizada com psicólogos atuantes em hospitais gerais, verificou-se que pouco se sabe sobre a metodologia dos CP em razão da falta de formação acadêmica. Esse déficit percebido nas pesquisas, bem como o panorama atual da saúde e as demandas emergentes, deixa clara a necessidade de existirem formas de capacitação mais eficientes acerca do cuidado de doentes fora de possibilidade de cura, para profissionais de saúde; e uma discussão aprofundada para a sociedade de uma forma geral.

\section{Considerações finais}

No Brasil, segundo a ANCP (2017b), as atividades relacionadas a $\mathrm{CP}$ ainda precisam ser regularizadas na forma de lei. Ainda predomina um enorme desconhecimento e muito preconceito relacionado a esses cuidados, principalmente entre profissionais de saúde, gestores hospitalares e poder judiciário. Se confunde CP com eutanásia e o preconceito com relação ao uso de opioides, para o alívio da dor, é enorme.

Por estas razões o conhecimento acerca dos CP é cada dia mais necessário para o bom exercício das práticas de saúde. A formação em nível técnico médio e superior, bem como a capacitação dos profissionais já em atuação, deve ser a maneira mais eficaz de fazer com que esses profissionais estejam mais preparados e sejam multiplicadores das práticas paliativistas, fortalecendo as políticas públicas e programas de humanização do cuidado em final de vida.

É impossível o aprofundamento nessa temática sem estabelecer relações tanto filosóficas, quanto teóricas e práticas com os programas e políticas públicas de saúde do SUS. Neste trabalho mostramos o quanto as políticas destinadas aos $\mathrm{CP}$ no Brasil são recentes e pouco trabalhadas em nível da formação profissional. Tal contexto só indica a necessidade de mais estudos na temática, de modo a deixar os profissionais de saúde mais preparados para cuidar da vida com humanismo e competência técnico-científica.

Por conseguinte, a formação competente nas práticas paliativistas se torna algo ainda mais urgente quando é observado o fenômeno de crescimento populacional no Brasil e no mundo, em que a expectativa de vida está cada vez maior e as tecnologias médicas se modernizando, de modo a aumentarem a longevidade humana. Por outro lado, muitas pessoas convivem durante anos e anos com doenças crônicas, e por isso, quanto mais "vivem" (ou morrem lentamente), mais precisam de cuidado humanizado.

O tema da morte e o morrer em suas relações com as práticas paliativistas precisam ser mais discutidos de forma crítica e reflexiva, almejando contribuir para a sua desmistificação e seu caráter tabu. Tal caráter, presente na maioria das pessoas e, inclusive entre os profissionais de saúde, faz com que esse assunto seja tratado como algo incômodo 
e distante, prejudicando as relações entre paciente, família, e para o próprio profissional. A convivência com a morte de pacientes com doenças terminais é constante nos hospitais, porém a maioria dos profissionais de saúde pouco discute o assunto com os doentes, e menos ainda com as famílias, pois têm dificuldade de tratar sobre o tema.

Evitar o tema da morte, por vezes, pode ser ainda mais prejudicial para os doentes, famílias, e também para o profissional, que entra em conflito consigo mesmo, acarretando sentimentos complexos e difi- culdades maiores de atuar em sua área. O processo de morte e do morrer precisa ser desmistificado, desvendado, estudado e refletido, de modo que as pessoas se preparem mais para esse momento, uma vez que é um fenômeno inevitável e natural da vida.

Concluímos nossas discussões, mas não encerramos o assunto. Nosso intuito também é provocar novos pesquisadores a refletirem sobre os $\mathrm{CP}$, levantar novos questionamentos que ainda precisam ser problematizados, tendo em vista a importância que o tema merece.

\section{Referências}

Academia Nacional de Cuidados Paliativos - ANCP (2017a). Onde existem. São Paulo, SP. Recuperado de http:// paliativo.org.br/ancp/onde-existem/

Academia Nacional de Cuidados Paliativos - ANCP (2017b). ANCP e cuidados paliativos no Brasil. Recuperado de http://paliativo.org.br/cuidados-paliativos/cuidados-paliativos-no-brasil/

Alves, R. (2003, outubro 12). Sobre a morte e o morrer. Folha de São Paulo, Opinião. Recuperado de http://wwwl. folha.uol.com.br/fsp/opiniao/fz1210200309.htm.

Alves, R. F., Melo, M., Andrade, S., \& Sousa, V. (2014). Saberes e práticas sobre cuidados paliativos segundo psicólogos atuantes em hospitais públicos. Psicologia, Saúde \&Doenças, 15(1), 78-96. https://doi.org/10.15309/14psd150108

Archanjo, J. V. L. (2010). Política nacional de humanização: Reafirmando os princípios do SUS (Dissertação de mestrado). Universidade Federal do Espírito Santo, Vitória, ES, Brasil.

Archanjo, J.V. L., \& Barros, M. E. B. (2009). Política nacional de humanização: Desafios de se construir uma "política dispositivo". Anais do Encontro Anual da ABRAPSO. Rio de Janeiro. RJ, 15. Recuperado de http://docplayer.com. br/13257665-Politica-nacional-de-humanizacao-desafios-de-se-construir-uma-politica-dispositivo.html

Barbi, M. Z. (2011). A inserção dos cuidados paliativos no sistema único de saúde (SUS) (Trabalho de conclusão de curso). 4 Estações Instituto de Psicologia, São Paulo, SP, Brasil.

Benevides, R., \& Passos, E. (2005). Humanização na saúde: Um novo modismo? Interface: Comunicação, Saúde, Educação, 9(17), 389-394. https://doi.org/10.1590/S1414-32832005000200014

Brasil. Ministério da Saúde. (2001). Programa nacional de humanização da assistência hospitalar. Brasília, DF: o autor.

Brasil. Ministério da Saúde. (2004). HumanizaSUS: Política nacional de humanização: A humanização como eixo norteador das práticas de atenção e gestão em todas as instâncias do SUS. Brasília, DF: o autor.

Brasil. Ministério da Saúde. (2010). Política nacional de humanização: Formação e intervenção. Brasília, DF: o autor.

Brasil. Ministério da Saúde. (2013). Portal da saúde: Melhor em casa, serviço de atenção domiciliar. Brasília, DF: o autor. Recuperado de http://portalsaude.saude.gov.br/index.php/cidadao/acoes-e-programas/melhor-em-casa

Carvalho, R. T., \& Parsons, H. A. (Org.). (2012). Manual de cuidados paliativos ANCP (2a ed. amp. atual.). São Paulo, SP: Academia Nacional de Cuidados Paliativos. Recuperado de http://biblioteca.cofen.gov.br/wp-content/uploads/2017/05/Manual-de-cuidados-paliativos-ANCP.pdf

Cohen, C., \& Gobbetti, G. J. (2001). Há autonomia para o paciente na UTI? Revista da Associação Médica Brasileira, 47(3), 169-197. https://doi.org/10.1590/S0104-42302001000300017

Crema, R. (2001). Prefácio. In: M. Hennezel, \& J. Y. Leloup, A arte de morrer: Tradições religiosas e espiritualidade humanista diante da morte na atualidade (4a ed.). Petrópolis, RJ: Vozes.

D’Avila, R. L., \& Ribeiro, D. C. (2011). A terminalidade da vida: Uma análise contextualizada da resolução CFM no 1.805/2006. In: R. D. Moritz (Org.), Conflitos bioéticos do viver e do morrer (pp. 187-238). Brasília, DF: Conselho Federal de Medicina. 
Deslandes, S. (2004). Análise do discurso oficial sobre a humanização da assistência hospitalar. Ciência \& Saúde Coletiva, 9(1), 7-14.

Floriani, C. A. (2009). Moderno movimento hospice: Fundamentos, crenças e contradições na busca da boa morte (Tese de Doutorado). Escola Nacional de Saúde Pública Sergio Arouca, Rio de Janeiro, RJ. Recuperado de https:// www.arca.fiocruz.br/handle/icict/2571

Floriani, C. A. (2011). Cuidados paliativos no Brasil: Desafios para sua inserção no sistema de saúde. In: F. S. Santos (Ed.), Cuidados paliativos: Diretrizes, humanização e alívio de sintomas (pp. 101-106). São Paulo, SP: Atheneu.

Floriani, C. A., \& Schramm, F. R. (2006). Cuidador do idoso com câncer avançado: Um ator vulnerado. Cadernos de Saúde Pública, 22(3), 527-534. https://doi.org/10.1590/S0102-311X2006000300007

Floriani, C. A., \& Schramm, F. R. (2007). Desafios morais e operacionais da inclusão dos cuidados paliativos na rede de atenção básica. Cadernos de Saúde Pública, 23(9), 2072-2080. https://doi.org/10.1590/S0102$311 X 2007000900015$

Floriani, C. A., \& Schramm, F. R. (2008). Cuidados paliativos: Interfaces, conflitos e necessidades. Ciência \& Saúde Coletiva, 13(2), 20123-2132. https://doi.org/10.1590/S1413-81232008000900017

Gil, A. C. (2002). Como elaborar projetos de pesquisa. São Paulo, SP: Atlas.

Junqueira, M. H. R., \& Kovács, M. J. (2008). Alunos de psicologia e a educação para a morte. Psicologia: Ciência e Profissão, 28(3), 506-519. https://doi.org/10.1590/S1414-98932008000300006

Kovács, M. J. (1999). Pacientes em estágio avançado da doença, a dor da perda e da morte. In: M. M. J. Carvalho (Org.), Dor: Um estudo multidisciplinar (2a ed.). São Paulo, SP: Summus.

Lago, P. M., Devictor, D., Piva, J. P., \& Bergouniou, J. (2007). Cuidados de final de vida em crianças: Perspectiva no Brasil e no mundo. Jornal de Pediatria, 83(2), 109-116. https://doi.org/10.1590/S0021-75572007000300013

Maciel, M. G. S. (2012). Organização de serviços de cuidados paliativos. In: R. T. Carvalho, \& H. A. Parsons (Org.), Manual de cuidados paliativos: ANCP (2a ed. amp. atual., pp. 94-110). São Paulo, SP: Academia Nacional de Cuidados Paliativos. Recuperado de http://biblioteca.cofen.gov.br/wp-content/uploads/2017/05/Manual-de-cuidados-paliativos-ANCP.pdf

Maciel, M. G. S., Rodrigues, L. F., Naylor, C., Bertega, R., Barbosa, S. M., Burlá, C. et al. (2006). Critérios de qualidade para os cuidados paliativos no Brasil. Rio de Janeiro, RJ: Diagraphic. Recuperado de http://www.paliativo.org. br/biblioteca_resultadobusca.php?sgeral=crit\%C3\%A9rios+de+qualidade+para+os+cuidados+paliativos\&button=Busca

Marengo, M. O., Flávio, D. A., \& Silva, R. H. A. (2009). Terminalidade de vida: Bioética e humanização em saúde. Revista Medicina, 42(3), 350-357. https://doi.org/10.11606/issn.2176-7262.v42i3p350-357

Matsumoto, D. Y. (2012). Cuidados paliativos: Conceitos, fundamentos e princípios. In: R. T. Carvalho, \& H. A. Parsons (Org.), Manual de cuidados paliativos: ANCP (2a ed. amp. atual., pp.23-30). São Paulo, SP: Academia Nacional de Cuidados Paliativos. Recuperado de http://biblioteca.cofen.gov.br/wp-content/uploads/2017/05/Manual-de-cuidados-paliativos-ANCP.pdf

Melo, M. O. (2012). Desafios da prática de psicólogos nos cuidados paliativos: Contributos para uma sistematização (Trabalho de Conclusão de Curso). Universidade Estadual da Paraíba, Campina Grande, PB. Recuperado de http://dspace.bc.uepb.edu.br/jspui/bitstream/123456789/314/1/PDF\%20\%20Myriam\%20de\%20Oliveira\%20 Melo.pdf

Montenegro, K. C. (2012). Cuidados paliativos e psicologia: A legitimação da alteridade como promoção da dignidade humana (Trabalho de Conclusão de Curso). Faculdade de Ciências da Educação e da Saúde, Brasília, DF. Recuperado de http://repositorio.uniceub.br/bitstream/123456789/2721/3/20706996.pdf

Mota, R. A., \& Martins, C. G. M. (2006). Papel dos profissionais de saúde na política da humanização hospitalar. Psicologia em Estudo, 11(2), 323-230. Recuperado de https://www.researchgate.net/publication/242245347_Papel_ dos_profissionais_de_saude_na_politica_de_humanizacao_hospitalar

Pasche, D., \& Passos, E. (2008). A importância da humanização a partir do sistema único de saúde. Revista Saúde Pública, 1(1), 92-100.

Pedreira, C. S. (2013). Assistência psicológica humanizada à pacientes oncológicos. Psicologia PT: O Portal dos Psicólogos, 1-14. Recuperado de http://www.psicologia.pt/artigos/textos/A0735.pdf 
Pessini, L., \& Bertachini, L. (2006). O que entender por cuidados paliativos. São Paulo, SP: Paulus.

Portaria no 19, de 03 de janeiro de 2002. Instituir, no âmbito do Sistema Único de Saúde, o Programa Nacional de Assistência à Dor e Cuidados Paliativos. Diário Oficial da União, 4 jan. 2002.

Resolução CFM no 1.805, de 28 de novembro, 2006. Na fase terminal de enfermidades graves e incuráveis é permitido ao médico limitar ou suspender procedimentos e tratamentos que prolonguem a vida do doente, garantindo-lhe os cuidados necessários para aliviar os sintomas que levam ao sofrimento, na perspectiva de uma assistência integral, respeitada a vontade do paciente ou de seu representante legal. Diário Oficial da União, 28 nov. 2006. Recuperado de http://www.portalmedico.org.br/resolucoes/cfm/2006/1805_2006.htm

Resolução CFM No 1.931, de 24 de setembro, de 2009. Aprova o Código de Ética Médica. Diário Oficial da União, 24 set. 2009.

Resolução CFM no 2.156, de 17 de novembro de 2016. Estabelece os critérios de admissão e alta em unidade de terapia intensiva. Diário Oficial da União, 18 nov. 2016. Recuperado de https://www.editoraroncarati.com.br/ v2/phocadownload/CFM_resolucao_2156_2016.pdf

Rodrigues, L. F. (2012). Modalidades de atuação e modelos de assistência em cuidados paliativos. In: R. T. Carvalho, \& H. A. Parsons, Manual de cuidados paliativos ANCP (pp. 86-93). São Paulo, SP: Academia Nacional de Cuidados Paliativos.

Rogers, C. R. (1983). Experiências em comunicação. In: C. R. Rogers, Um jeito de ser (pp. 3-16). São Paulo, SP: EPU.

Santos, F. S. (Ed.). (2011). Cuidados paliativos: Diretrizes, humanização e alívio de sintomas. São Paulo, SP: Atheneu.

Saunders, C. (2004). Foreword. In: D. Doyle, G. Hanks, N. Cherny, K. Calman, (Eds.), Oxford textbook of palliative medice (3a ed., pp. 17-20). Oxford: Oxford University.

Severino, A. J. (1993). Metodologia do trabalho científico. São Paulo, SP: Cortez.

Silva, M., \& Moreira, M. C. (2010). Desafios à sistematização da assistência de enfermagem em cuidados paliativos oncológicos: Uma perspectiva da complexidade. Revista Eletrônica de Enfermagem, 12(3), 483-490. https://doi. org/10.5216/ree.v12i3.7274

Sociedad Española de Cuidados Paliativos - Secpal. (2011). Guia de cuidados paliativos. Madri: o autor. Recuperado de http://www.secpal.com//Documentos/Paginas/guiacp.pdf

Tonetto, L. M., \& Rech, T. (2001). Lidar com a terminalidade: Um desafio para o psicólogo. Psico, 32(1), 5-6.

Unidade de Cuidados - UNIC. (2009). Manual de cuidados paliativos em pacientes com câncer. Rio de Janeiro, RJ: Universidade Aberta à Terceira Idade.

Waldow, V. R., \& Borges, R. F. (2011). Cuidar e humanizar: Relações e significados. Acta Paulista de Enfermagem, 24(3), 414-418. http://doi.org/10.1590/S0103-21002011000300017

World Health Organization - WHO. (1990). Cancer pain relief and palliative care. Genebra: o autor.

World Health Organization -WHO. (2002). OMS Definição de cuidados paliativos. Genebra: o autor. Recuperado de http://www.who.int/cancer/palliative/definition/en/

\section{Railda Sabino Fernandes Alves}

Professora Doutora do Departamento de Psicologia e do Programa de Pós-Graduação em Psicologia da Saúde da Universidade Estadual da Paraíba, Campina Grande - PB. Brasil.

E-mail: raildafernandesalves@gmail.com

(iD) https://orcid.org/0000-0001-5093-1952

Elizabeth Cristina Nascimento Cunha

Psicóloga do Centro de Referência de Assistência Social, Remígio - PB. Brasil.

E-mail: elizabethcristina_nc@hotmail.com

(iD https://orcid.org/0000-0002-6730-3351 


\section{Gabriella Cézar Santos}

Psicóloga clínica. Mestra em Psicologia da Saúde pela Universidade Estadual da Paraíba, Campina Grande - PB. Brasil.

E-mail: gabriellacezar@hotmail.com

(iD) http://orcid.org/0000-0003-0086-3626

\section{Myriam Oliveira Melo}

Psicóloga do Instituto Federal de Educação, Ciência e Tecnologia do Estado do Pará (IFPA). Mestra em Psicologia da Saúde pela Universidade Estadual da Paraíba, Campina Grande - PB. Brasil.

E-mail: myriamoliveiramelo@hotmail.com

(iD http://orcid.org/0000-0002-1341-710X

Endereço para envio de correspondência:

Avenida Mar a Sibéria, 117, apto 902. Intermares.

Cabedelo. PB - Brasil, Cep: 58102-071

Recebido 25/09/2017

Aceito 20/03/2018

Received 09/25/2017

Approved 03/20/2018

Recibido 25/09/2017

Aceptado 20/03/2018

Como citar: Alves, R. S. F, Cunha, E. C. N., Santos, G. C., \& Melo, M. O. (2019). Cuidados paliativos: Alternativa para o cuidado essencial no fim da vida. Psicologia: Ciência e Profissão, 39, 1-15. https://doi.org/10.1590/1982-3703003185734

How to cite: Alves, R. S. F., Cunha, E. C. N., Santos, G. C., \& Melo, M. O. (2019). Palliative care: Alternative to essential care at the end of life. Psicologia: Ciência e Profissão, 39, 1-15. https://doi.org/10.1590/1982-3703003185734

Cómo citar: Alves, R. S. F., Cunha, E. C. N., Santos, G. C., \& Melo, M. O. (2019). Cuidados paliativos: Alternativa para el cuidado esencial en el fin de la vida. Psicologia: Ciência e Profissão, 39, 1-15. https://doi.org/10.1590/1982-3703003185734 